\title{
Addiction of pancreatic cancer cells to zinc-finger transcription factor ZIC2
}

\author{
Shingo Inaguma ${ }^{1}$, Hideaki Ito ${ }^{1}$, Miho Riku${ }^{1}$, Hiroshi Ikeda ${ }^{1}$ and Kenji Kasai ${ }^{1}$ \\ ${ }^{1}$ Department of Pathology, Aichi Medical University School of Medicine, Nagakute, Aichi, Japan \\ Correspondence to: Kenji Kasai, email: kkasai@aichi-med-u.ac.jp \\ Shingo Inaguma, email: inaguma@aichi-med-u.ac.jp \\ Keywords: ZIC2, GLI1, ANXA8, FGFR3, apoptosis \\ Received: June 06, $2015 \quad$ Accepted: July 03, $2015 \quad$ Published: July 22, 2015
}

This is an open-access article distributed under the terms of the Creative Commons Attribution License, which permits unrestricted use, distribution, and reproduction in any medium, provided the original author and source are credited.

\section{ABSTRACT}

Activity of GLI transcription factors of Hedgehog signaling is key for various cancer cell properties, especially in pancreatic ductal adenocarcinoma (PDAC). Zincfinger transcriptional regulators ZIC1 to ZIC5 of ZIC gene family were demonstrated to associate with GLI to increase the nuclear accumulation and transcriptional activity of GLI. Notwithstanding this supportive role for GLI-dependent transcription, it was not fully understood whether ZIC plays an independent role in cancer cell biology. Here, we found that ZIC2 is indispensable in the regulation of PDAC cell apoptosis. We found that human PDAC cell lines uniquely express ZIC2. ZIC2 knockdown induced PDAC cell apoptosis; conversely, ZIC2 over-expression enhanced the cellular proliferation. Through a comprehensive screening, we identified fibroblast growth factor receptor 3 (FGFR3) and ANNEXIN A8 (ANXA8) as genes up-regulated by ZIC2 in PDAC cells. The forced expression of these two genes cooperatively rescued the apoptosis of ZIC2knockdown cells. Immunohistochemical analyses further supported the correlation of ZIC2 expression and these genes in human pancreata harboring PDAC. Intriguingly, the ZIC2-mediated up-regulation of FGFR3 and ANXA8 was indicated to be GLI -independent. This evidence highlights the indispensable role of ZIC2 in regulating cellular proliferation and apoptosis during PDAC development and suggests a potential therapeutic target for PDAC.

\section{INTRODUCTION}

The members of the ZIC gene family, vertebrate homologues of the Drosophila odd-paired gene, encode a nuclear protein harboring a $\mathrm{C} 2 \mathrm{H} 2$ type of zinc-finger domain that shows a notable homology to the GLI transcription factors GLI1-3 of the Hedgehog pathway[1] [2]. ZIC is mainly expressed in the developing or mature central nervous system (CNS) in a spatiotemporally restricted manner, and ZIC2 mutation causes various developmental anomalies, including holoprosencephaly, which is also linked to the mutations of the Hedgehog pathway-related genes [3].

GLI1 and GLI2 are over-expressed and thought to participate in the development and progression of various cancers, especially pancreatic ductal adenocarcinoma (PDAC) [4]. It was experimentally confirmed that GLI1 is indispensable for the oncogenic mutant type of KRASdependent transformation of pancreatic epithelium in a genetically-modified mouse model [5-7] and that GLI1 is essential for the survival and maintenance of the transformed phenotype of human PDAC cell lines. Indeed, GLI1 was revealed to up-regulate the expression of a variety of genes crucial for many cancer cell properties [8-12].

A previous study using the electrophoretic mobility shift assay (EMSA) and in vitro binding analysis reported that GLI and ZIC bound to similar DNA sequences, indicating a close relationship between ZIC and GLI in the regulation of downstream target genes [2]. Moreover, ZIC was reported to associate with GLI and enhance the nuclear accumulation and transcriptional activity of GLI proteins. $[13,14]$ Therefore, it might be possible that the ZIC-dependent regulation of GLI target genes is involved 
in the cell properties of PDAC; however, whether ZIC is expressed in PDAC cells and the role of ZIC, if expressed, have not been addressed.

In the present study, we first identified $Z I C 2$ as a unique member of the ZIC gene family expressed in PDAC cells. ZIC2 knockdown lead to PDAC cell apoptosis and, in turn, ZIC2 over-expression enhanced PDAC cell proliferation. We found that ZIC2 up-regulated the expression of FGFR 3 and $A N X A 8$, and these two genes cooperatively rescued the apoptotic cell death of $Z I C 2$ knockdown cells. Interestingly, we revealed that the upregulation of FGFR 3 and $A N X A 8$ by ZIC2 occured in a GLI-independent manner. Our results uncovered the indispensable and GLI-independent role of ZIC2 in the regulation of PDAC cell apoptosis.

\section{RESULTS}

\section{ZIC2 regulates the apoptotic cell death of PDAC cells}

We first examined the expression of the ZIC gene family (ZIC1-5) in immortalized normal pancreatic epithelial cells, hTERT-HPNE (HPNE) cells and human
PDAC cell lines. Reverse transcription (RT)-PCR analysis revealed that none of the $Z I C$ members were expressed in HPNE cells while the expression of all members of the family was detected in a control material (human cerebellum cDNA). We found, however, that all of the human PDAC cell lines we tested uniquely expressed ZIC2 (Figure 1A). Compared with three independent datasets in the Oncomine database [15-17], we again found that ZIC2 expression was dominantly increased in PDAC cells rather than normal pancreatic tissue (Figure 1B). Therefore, we hypothesized that ZIC2 may be involved in the PDAC development. To clarify this, we examined the effect of ZIC2 knockdown in the human PDAC cell lines PANC-1 and KP-4 by the transfection of specific siRNA (for knockdown validation, see Figure 2C). We found that the transfection of ZIC2-specific siRNA (siZIC2-1 and siZIC2-2) but not control siRNA (siControl) suppressed the cellular proliferation of both cell lines (Figure 2A). Cell cycle analysis revealed that ZIC2 knockdown reduced the fraction of cells in the G0/G1 phase and increased the fraction of cells in the sub-G1 phase (representative data in Figure 2B, upper panels; the statistical analysis from three independent experiments in Figure 2B, lower table). Additionally, ZIC2 knockdown concordantly increased the amount of cleaved PARP (Figure 2C). These results indicated the indispensable role of ZIC2 in

A

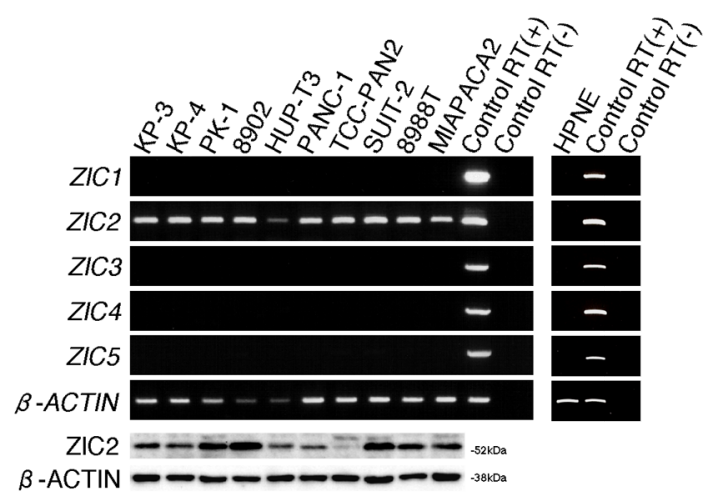

B

\begin{tabular}{|l|c|c|c|c|c|}
\hline & ZIC1 & ZIC2 & ZIC3 & ZIC4 & ZIC5 \\
\hline Grützmann R, et al. & & 9.3 & & 1.7 & N.D. \\
\hline Pei H, et al. & & 1.6 & & & \\
\hline Badea L, et al. & & 1.4 & & & \\
\hline
\end{tabular}

Figure 1: ZIC2 is uniquely expressed in PDAC cells. A, RT-PCR and immunoblot analysis of the human PDAC cell lines and the immortalized normal pancreatic epithelial cell line HPNE. Normal fetal cerebellar RNAs with or without the RT reaction were used as controls. B, The relative expression of the ZIC gene family in PDAC tissue normalized to normal tissue. The data sets were drawn from the Oncomine database. The original publications are listed in the references. Note that Grützmann R. et al. [16]. used microdissected samples for cDNA array analysis. 
A
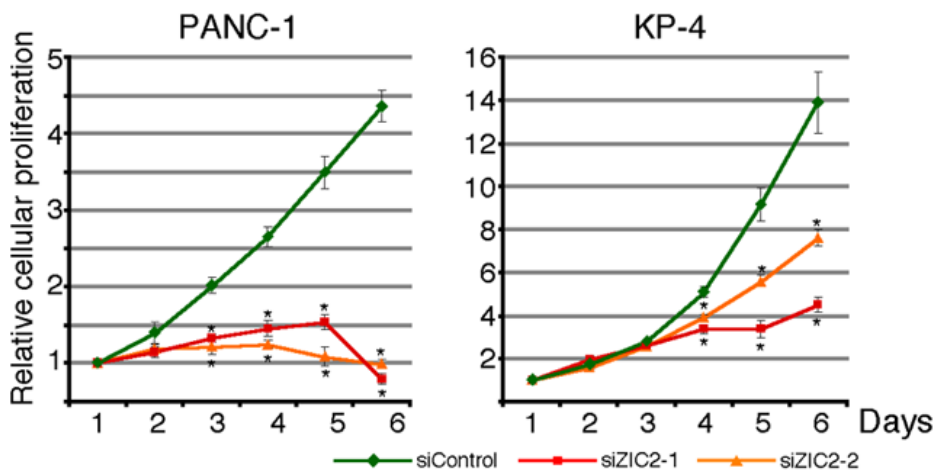

B
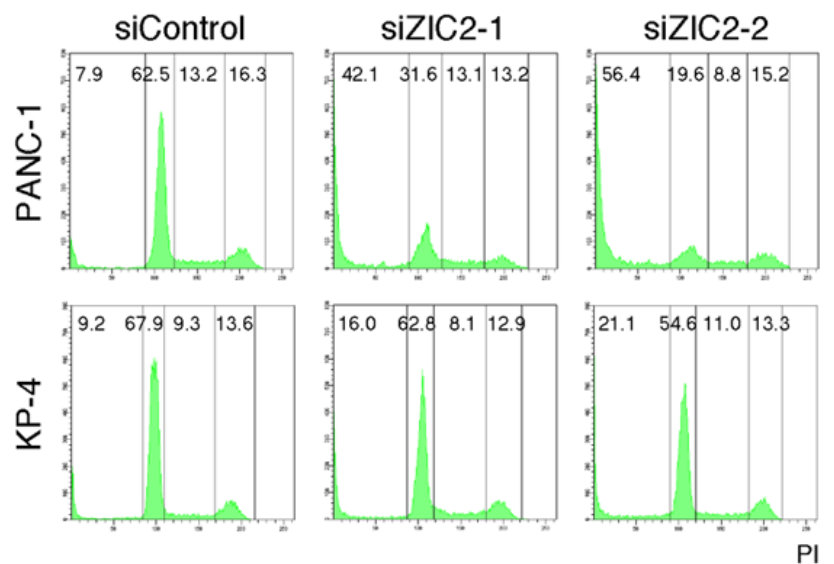

Ratio of sub-G1 phase (\%)

\begin{tabular}{|l|c|c|c|}
\hline & siControl & siZIC2-1 & siZIC2-2 \\
\hline PANC-1 & $9.2 \pm 0.6$ & $42.0 \pm 1.1^{*}$ & $56.8 \pm 0.9^{*}$ \\
\hline KP-4 & $9.3 \pm 0.3$ & $17.0 \pm 0.9^{*}$ & $21.7 \pm 0.7^{*}$ \\
\hline
\end{tabular}

*:p<0.01 (v.s. siControl)

C

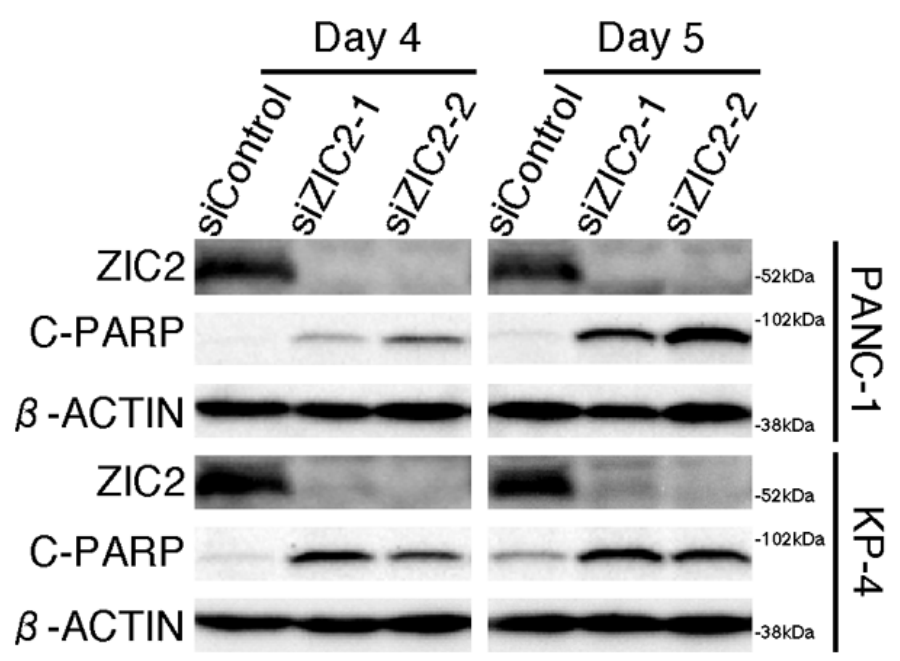

Figure 2: ZIC2 regulates apoptotic cell death of PDAC cells. A and B, The proliferation curves and cell cycle analysis of PDAC cells transfected either ZIC2-specific (siZIC2-1, siZIC2-2) or control (siControl) siRNAs. The data represent the mean \pm SD from three independent experiments. C, immunoblot analysis of PDAC cells transfected with siRNAs. 
regulating the apoptotic cell death of PDAC cells. Next, we analyzed the effects of ZIC2 using newly established cell lines from the human PDAC cell line PANC-1: these derivatives show the Tet-regulated expression (Tet-off) of ZIC2 (Supplementary Figure S1A). As a result, we found that the forced expression of ZIC2 enhanced the G1-S transition and cellular proliferation (Supplementary Figure $\mathrm{S} 1 \mathrm{~B}$ and $\mathrm{C}$ ). It was reported that ZIC2 over-expression induces anchorage-independent growth and transformed foci of mouse embryonic fibroblasts (MEF) [18]. Indeed, we also observed that lentiviral-transduced ZIC2 enhanced cellular proliferation (Supplementary Figure S1D) and the anchorage-independent growth of HPNE cells (Supplementary Figure S1E). Unfortunately, neither ZIC2- nor control (LacZ)-transduced HPNE cells were transplantable to NOD/SCID mice in our experimental conditions (data not shown): therefore, these results indicated that ZIC2 over-expression led to the cellular proliferation of the pancreatic duct epithelium, while we could not conclude at this moment that it could transform the pancreatic duct epithelium.

A

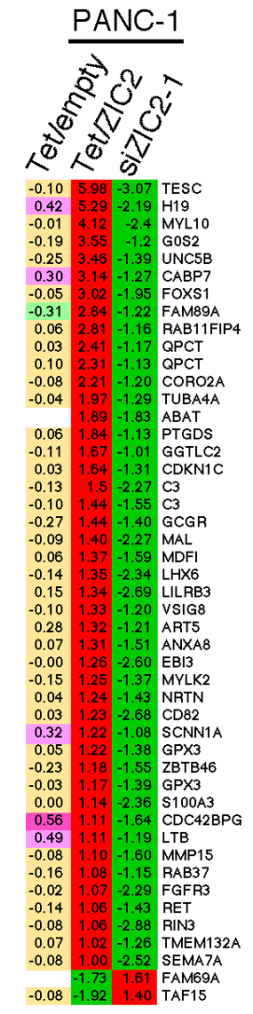

B

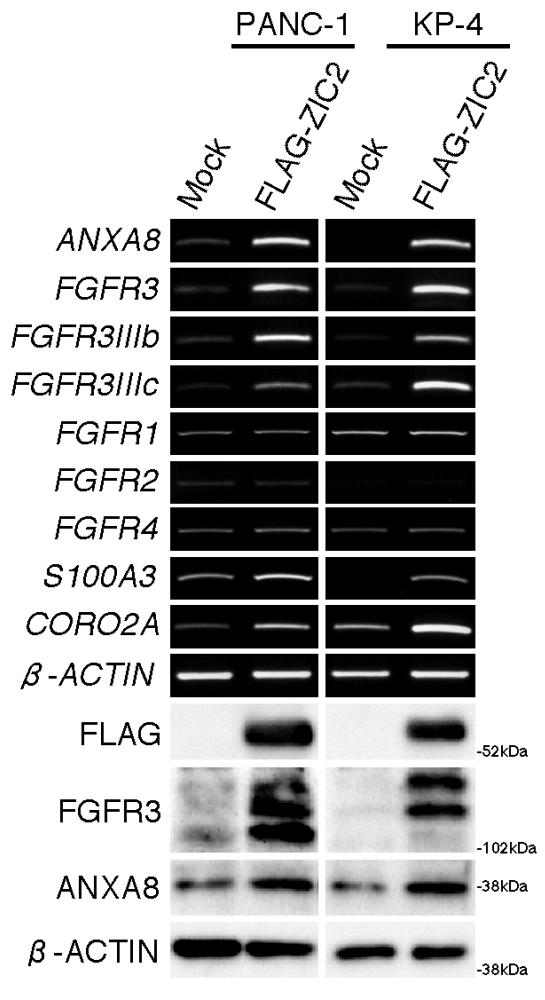

\section{ZIC2 up-regulates ANXA8 and FGFR3}

To date, ZIC2 over-expression was reported to impact the clinical course and prognosis of patients harboring ovarian cancer [18], endometrial cancer [19] and oral squamous cell carcinoma [20], while the molecular mechanism of disease progression was not revealed. Given that ZIC2 is a transcription factor, we hypothesized that ZIC2 might regulate the expression of genes that are responsible for PDAC cell proliferation and apoptosis. To investigate this possibility, we performed three sets of expression (cDNA) microarray analyses and identified the differentially-expressed genes as follows: from the first set, the genes changed by ZIC2 knockdown in PANC-1 cells; from the second set, the genes changed by the elimination of DOX in PANC-1 $1^{\text {Tet/ZIC2 }}$ clone1; and from the third set, the genes that were "left unchanged" after the elimination of DOX in control PANC-1 ${ }^{\mathrm{Tet} /}$ empty to eliminate the artificial change by DOX itself. By comparing the chosen genes from these three independent analyses, we identified 43 up-regulated genes, as well as 2 down-regulated genes, as candidate genes downstream of

C
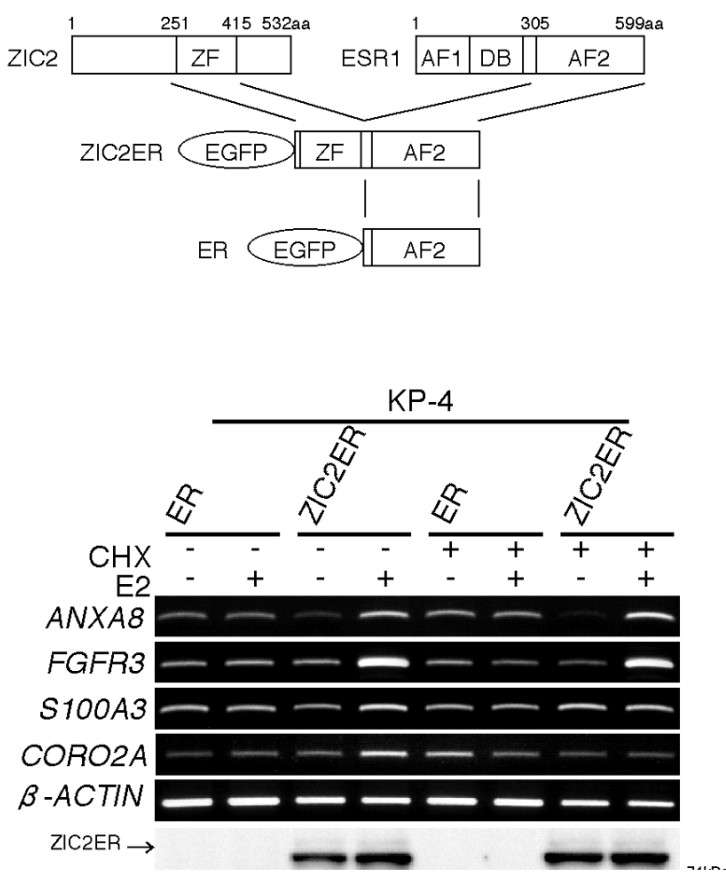

Figure 3: ZIC2 up-regulates ANXA8 and FGFR3. A, Comparative presentation of three sets of the expression microarray analysis. Tet/empty, the genes of PANC-1 ${ }^{\text {Tet/empty }}$ cells not changed by the DOX elimination; Tet/ZIC2, the genes of PANC-1 ${ }^{\text {Tet ZIC2 }}$ cells changed by the DOX elimination; siZIC2-1, the genes of PANC-1 cells changed by siZIC2-1-treanfection, which was normalized by siControl transfection. The numbers are on the $\log 2$ scale. B, RT-PCR and immunoblot analyses of PDAC cells transiently transfected with either a FLAG-tagged ZIC2 expression vector or an empty (Mock) vector. C, Schematic of the transgenes (upper panel) and RT-PCR / immunoblot analysis (lower panel) of KP-4 cells, which were transiently transfected with either ZIC2ER or a control ER vector. $C H X$, cycloheximide; E2, $\beta$-estradiol. 
ZIC2 (Figure 3A; for a validation, Supplementary Figure $\mathrm{S} 2 \mathrm{~A}$ ). From the list, we chose $A N X A 8, F G F R 3, S 100 A 3$ and $C O R O 2 A$ and confirmed that their expression levels were indeed up-regulated by the transient transfection of the FLAG-tagged ZIC2 (FLAG-ZIC2) expression vector in either PANC-1 or KP-4 cells (Figure 3B).

Next, we transiently transfected KP-4 cells with either the chimeric gene ZIC2ER, which consisted of the EGFP-tagged DNA-binding (zinc-finger) domain of human ZIC2 and the AF2 domain of the mouse Esr1, or its control EGFP-tagged AF2 domain (ER; illustration of the chimeric genes in Figure $3 \mathrm{C}$, upper panel). The former, not the latter, was expected to rapidly up-regulate the expression of downstream genes upon treatment with $\beta$-estradiol (E2), as we previously reported $[9,10]$. Indeed, E2 treatment up-regulated the expression of these four genes only in the ZIC2ER-transfected cells (Figure 3C). Furthermore, the up-regulation of $A N X A 8$ and FGFR3 but not $S 100 A 3$ and $C O R O 2 A$ was also observed even in the presence of cycloheximide (CHX), indicating that the upregulation of $A N X A 8$ and FGFR3 was not dependent on new protein synthesis (Figure $3 \mathrm{C}$ ). We also confirmed the up-regulation of $A N X A 8$ and FGFR3 in ZIC2-transduced
HPNE cells (Supplementary Figure S2B). This evidence indicated that $A N X A 8$ and FGFR3 were downstream of ZIC2.

The FGFR gene family of receptor tyrosine kinases is known to regulate the cellular proliferation of a variety of cancers, including PDAC [21]. $A N X A 8$ is a member of the super gene family encoding calciumand phospholipid-binding proteins, which participate in various cellular functions, including calcium signaling, vesicle trafficking, cell division, growth regulation and apoptosis [22]. Recently, $A N X A 8$ was reported to be up-regulated in PDAC tissue [23] and to suppress the apoptotic cell death of PDAC cells [24]. Therefore, we examined FGFR 3 and $A N X A 8$ as a downstream target of ZIC2 in PDAC cells.

Recently a comprehensive analysis of the ZIC2binding domain revealed that ZIC2 preferentially binds to a transcriptional enhancer, the majority of which are located outside of the transcription start site [25]. Indeed, we found that ZIC2 did not activate the luciferase reporter constructs harboring up to $4 \mathrm{~kb}$ DNA fragments of the 5' flanking sequence from transcription start sites of FGFR3 and $A N X A 8$ genes (data not shown), leaving the possibility
A
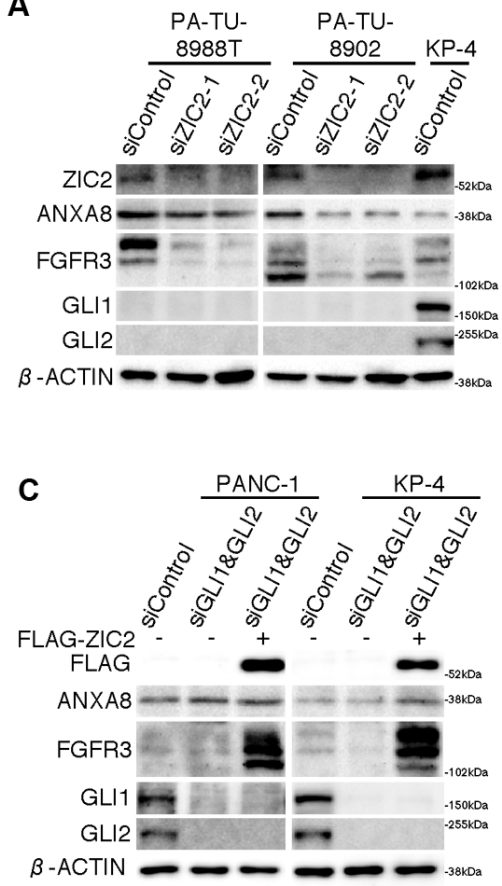

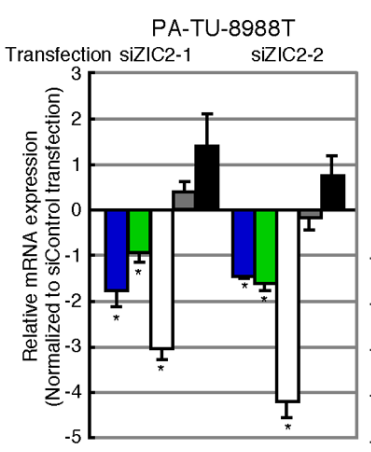

PANC-1

Transfection siGLI1\&2+mock siGLI1\&2+ZIC2

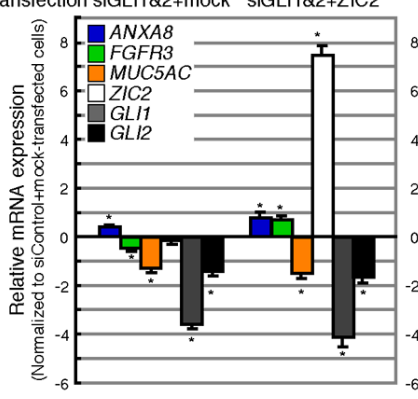

B
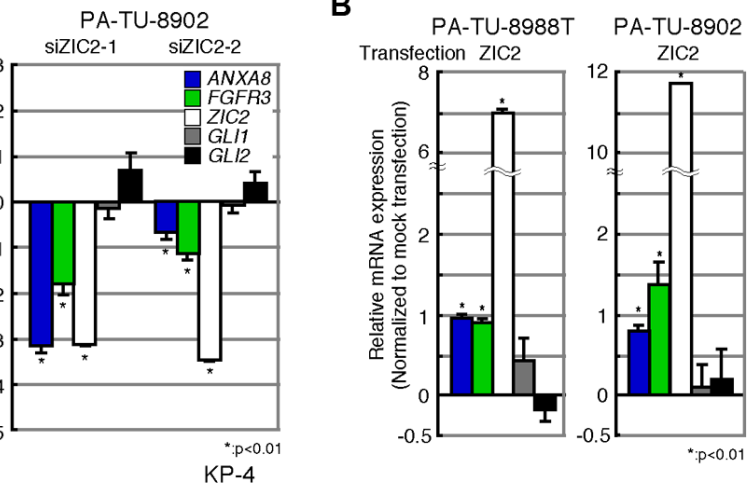

siGLI1\&2+mock siGLI1\&2+ZIC2

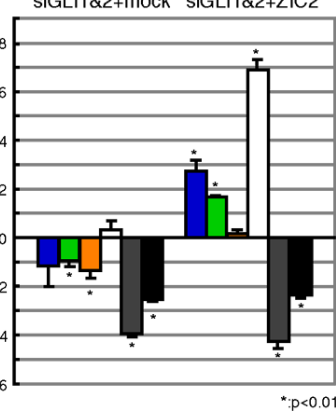

Figure 4: Up-regulation of ANXA8 and FGFR3 is GLI -independent. A, Immunoblot (left) and qRT-PCR (right) analyses of GLI low-expressing PDAC cells (PA-TU-8988T and PA-TU-8902) transiently transfected with indicated siRNAs. The immunoblot of GLI high-expressing KP-4 cells is also shown as a comparison. The RT-PCR data were normalized to that of siControl transfectants and are shown on the $\log 2$ scale. Columns, the mean values of three independent experiments; bars, SD. B, qRT-PCR analysis of GLI low-expressing PDAC cells transiently transfected with either a FLAG-ZIC2 expression vector or an empty (Mock) vector. The data were normalized to that of mock vector transfectants and are shown on the $\log 2$ scale. Columns, the mean values of three independent experiments; bars, SD. C, Immunoblot (left) and qRT-PCR (right) analyses of GLI high-expressing PDAC cells (PANC-1 and KP-4) transiently transfected with either siRNAs for a GLI1/2-double knockdown (siGLI1\&siGLI2) or a control siRNA (siControl) in conjunction with FLAG-ZIC2 (+) or a mock control vector (-). In qRT-PCR analysis, the data were normalized to that of siControl plus a mock control expression vector transfectants and are shown on the $\log 2$ scale. Columns, the mean values of three independent experiments; bars, SD. 
that the distant enhancer of the FGFR3 and $A N X A 8$ genes might be responsible for ZIC2-dependent up-regulation.

The other members of FGFR, such as FGFR1, FGFR2 and FGFR4, were not changed by FLAG-ZIC2 transfection (Figure $3 \mathrm{~B}$ ). The up-regulation of both RET and its ligand Neurtrin (NRTN) was intriguing (Figure 3A and Supplementary Figure S2A), but the expression of RET and its co-receptor GFRAl were detected only in a few limited PDAC cell lines (Supplementary Figure S3). Therefore, we considered that the NRTN-RET axis might not be a major pathway in PDAC cells.

\section{Up-regulation of ANXA8 and FGFR3 is GLI- independent}

It was reported that ZIC2 interacts with GLI1 to enhance the nuclear translocation and transcription activity of GLI1 $[13,14]$, indicating a supportive role of
ZIC2 in GLI1-target gene expression. Thus, we examined whether ZIC2 up-regulates $A N X A 8$ and FGFR3 in a GLIdependent manner. First, we employed human PDAC cell lines PA-TU-8988T and PA-TU-8902, which expressed GLI1 and GLI2 at very low levels (Figure 4A, left panel). The expression of $A N X A 8$ and FGFR3 was reduced by ZIC2-knockdown without an obvious suppression of GLI1 and GLI2 (Figure 4A) and was conversely increased by a transient transfection of FLAG-ZIC2 (Figure 4B). Next, using PANC-1 and KP-4, which highly expressed GLI1 and GLI2 (Figure 4C, left panel), we found that the ZIC2 transfection further increased the expression of $A N X A 8$ and FGFR3 in the context of double-knockdown of GLI1 and GLI2 (Figure 4C). The change of ANXA8 and FGFR3 was in contrast to $M U C 5 A C$, which we previously reported as a direct target gene of GLI1 and GLI2 [9]. This evidence suggested that ZIC2 up-regulates $A N X A 8$ and FGFR3 in a GLI-independent manner.
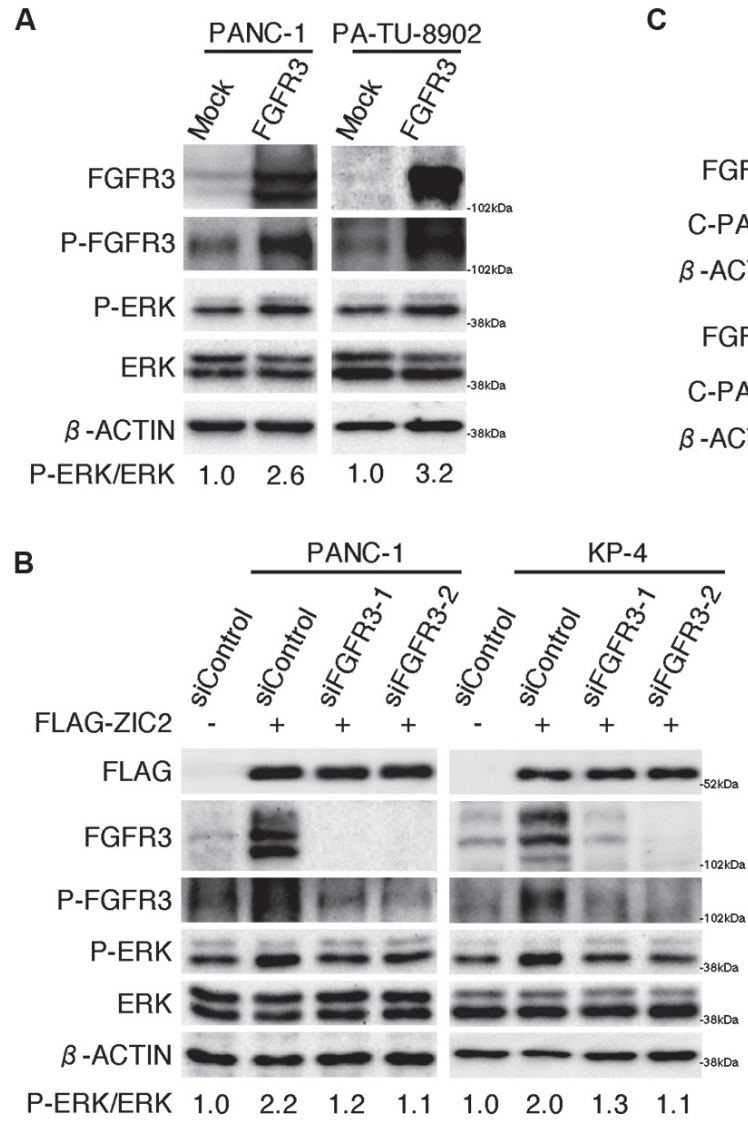
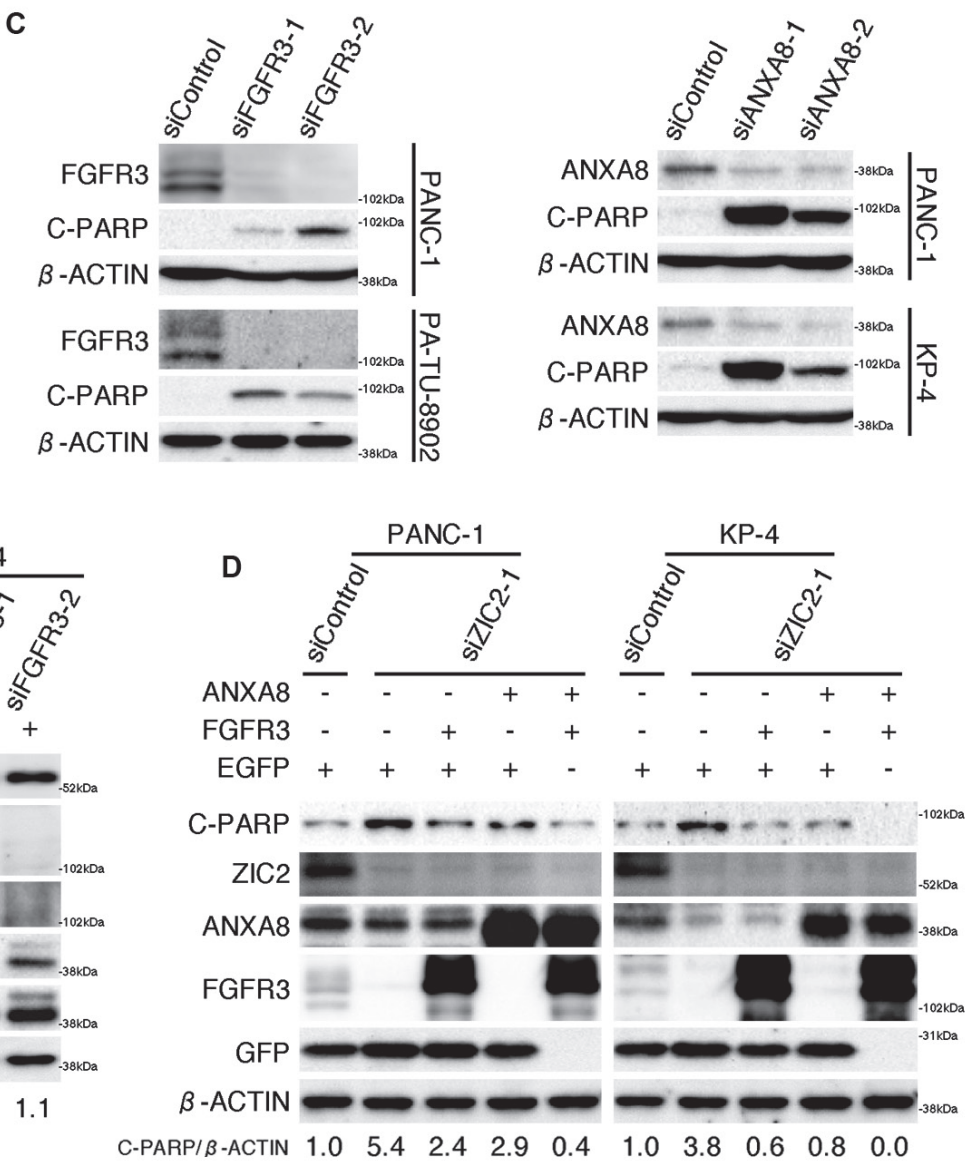

Figure 5: ANXA8 and FGFR3 cooperatively rescue PDAC cells from apoptotic cell death triggered by ZIC2 knockdown. A, Immunoblot analysis of PDAC cells transiently transfected with either an FGFR3-IIIb variant of the human FGFR3 expression vector (FGFR3) or an empty (Mock) control vector. The ratio of phosphorylated ERK versus ERK protein was semi-quantified using NIH Image software. B, Immunoblot analysis of PDAC cells transiently transfected with either FGFR3-specific siRNAs (siFGFR3-1, siFGFR3-2) or a control siRNA (siControl) in conjunction with either FLAG-ZIC2 (+) or an empty (Mock) vector (-). C, immunoblot analysis of PDAC cells transiently transfected with either FGFR3-specific, ANXA8-specific (siANXA8-1, siANXA8-2) or control siRNAs. D, Immunoblot analysis of PDAC cells transfected with either siZIC2-1 or siControl in conjunction with the indicated expression vectors (EGFP, FGFR3 and ANXA8). The DNA amounts of transfected expression vectors were equalized using the EGFP expression vector. 


\section{ZIC2 phosphorylation of ERK}

FGFR3-dependent

While FGFR1, FGFR2 and FGFR4 are frequently over-expressed in PDAC and are well recognized to enhance cellular proliferation via several downstream pathways, including the RAS-MAPK-ERK pathway [21], the effect of FGFR3 in PDAC cells is controversial. Lafitte et al. reported that the forced expression of $F G F R 3$ enhanced the cellular proliferation in a vimentinrich "mesenchymal" type of PDAC cell via the MAPK pathway, while it suppressed the cellular proliferation in an E-cadherin-rich "epithelial" type of PDAC cell [26]. We found that splicing variants of FGFR3 ("mesenchymal" type PDAC-expressing FGFR3-IIIb and "epithelial" type PDAC-expressing FGFR3-IIIc) were both up-regulated by FLAG-ZIC2 transfection (Figure 3B). By the transient transfection of the expression vector of FGFR3-IIIb splice variant of human $F G F R 3$ gene, we also found that the FGFR3 up-regulation increased the phosphorylation of FGFR3 itself and ERK in both "epithelial" type PATU-8902 and "mesenchymal" type PANC-1 (Figure 5A; Supplementary Figure S4 for E-cadherin / vimentin expression profile of PDAC cell lines), indicating the impact of FGFR3 on the MAPK pathway in our experimental system.

Next, to examine the effect of ZIC2 on the FGFR3MAPK pathway, we transiently transfected PANC-1 and KP-4 with the FLAG-ZIC2 expression vector in conjunction with either $F G F R 3$-specific siRNAs or a control siRNA. We found that the forced expression of ZIC2 enhanced the phosphorylation of FGFR3 and ERK, coincident with the increased expression of FGFR3. We also found that the ZIC2-enhanced phosphorylation of ERK returned to the original levels by FGFR 3 knockdown (Figure 5B). We cannot say, however, whether the expression of $F G F 1$ and $F G F 2$ (Figure 3B), ligands of FGFR, or a "molecular crowding" of FGFR3 might intrinsically stimulate FGFR3 signaling [21], this question should be investigated in the future.

\section{ANXA8 and FGFR3 cooperatively rescue PDAC cells from apoptotic cell death triggered by $\mathrm{ZIC} 2$ knockdown}

The activation of the MAPK-ERK pathway has been known to regulate PDAC apoptosis [27]. Indeed, we found that FGFR3 knockdown increased cleaved PARP levels in both PDAC cell lines (Figure 5C, left panel). Recently, ANXA8 was reported to be up-regulated in PDAC tissue [23] and to suppress PDAC cell apoptosis [24]. In agreement with this result, we also found that ANXA8 knockdown led to increased cleaved PARP levels (Figure 5C, right panel). Given that ZIC2 knockdown led to apoptotic cell death, we considered the possibility that FGFR3 or ANXA8 prevents the apoptotic cell death induced by ZIC2 -knockdown. We transiently transfected PANC-1 and KP-4 with the expression vectors for $A N X A 8$ or FGFR3 in conjunction with siRNA for ZIC2 -knockdown. We found that the forced expression of either ANXA 8 or FGFR 3 alone could slightly but not completely reduce the cleaved PARP induced by ZIC2-knockdown, while co-transfection of these two genes showed a better suppression of cleaved PARP (Figure 5D). This evidence suggested that ZIC2 over-expression regulated apoptotic cell death of PDAC cells through the up-regulation of ANXA8 and FGFR3.

\section{ZIC2 expression correlates with ANXA8 and FGFR3 expression and Ki-67 labeling index in pancreatic tissue}

Finally, we examined the expression of ZIC2, ANXA8, FGFR3 and the cell proliferation marker Ki67 in PanIN and PDAC by immunohistochemistry. To evaluate the expression of ZIC2, ANXA8 and FGFR3, we used a semi-quantitative scoring of the staining intensity on a three-tiered scale (negative, 0 ; weak, 1 ; strong, 2) and statistical analysis as previously shown $[9,10]$. ZIC2 was not detectable in the normal pancreatic duct, but was faintly expressed in the low-grade PanIN, then increased in the high-grade PanIN and PDAC cells (Figure 6A and Supplementary Table S1). The expression levels of ANXA8 and FGFR3 were also faintly detectable in the normal duct; however, their expression gradually increased along with ZIC2 expression (Figure 6A and Supplementary Tables S2 and S3). Spearman's rank correlation analysis revealed positive correlations between ZIC2 and its target genes (Figure 6B, 6C). The Ki-67 labeling index was $0.5-3 \%$ in the normal duct and lowgrade PanIN; then it increased to $9-19 \%$ and $34 \%$ in the high-grade PanIN and PDAC, respectively (Figure 6A and Supplementary Table S4). Spearman's rank correlation analysis also revealed positive correlations between ZIC2 expression and Ki-67 labeling index (Figure 6D).

\section{DISCUSSION}

The dysregulated expression of GLI1 and GLI2 plays a crucial role in the development and progression of many types of human cancers, including PDAC [5-7]. To fully activate the transcription activity of GLI, a multistep molecular mechanism is required [28]: for instance, we reported that the SIL / STIL-mediated derepression from Suppressor-of-Fused is involved during PDAC development [29]. It was previously reported that ZIC and GLI bind to similar DNA sequences in vitro [2] and that ZIC enhances the nuclear accumulation and transcriptional activity of GLI proteins $[13,14]$, suggesting a supportive role for ZIC in the activation of the GLI target expression. 


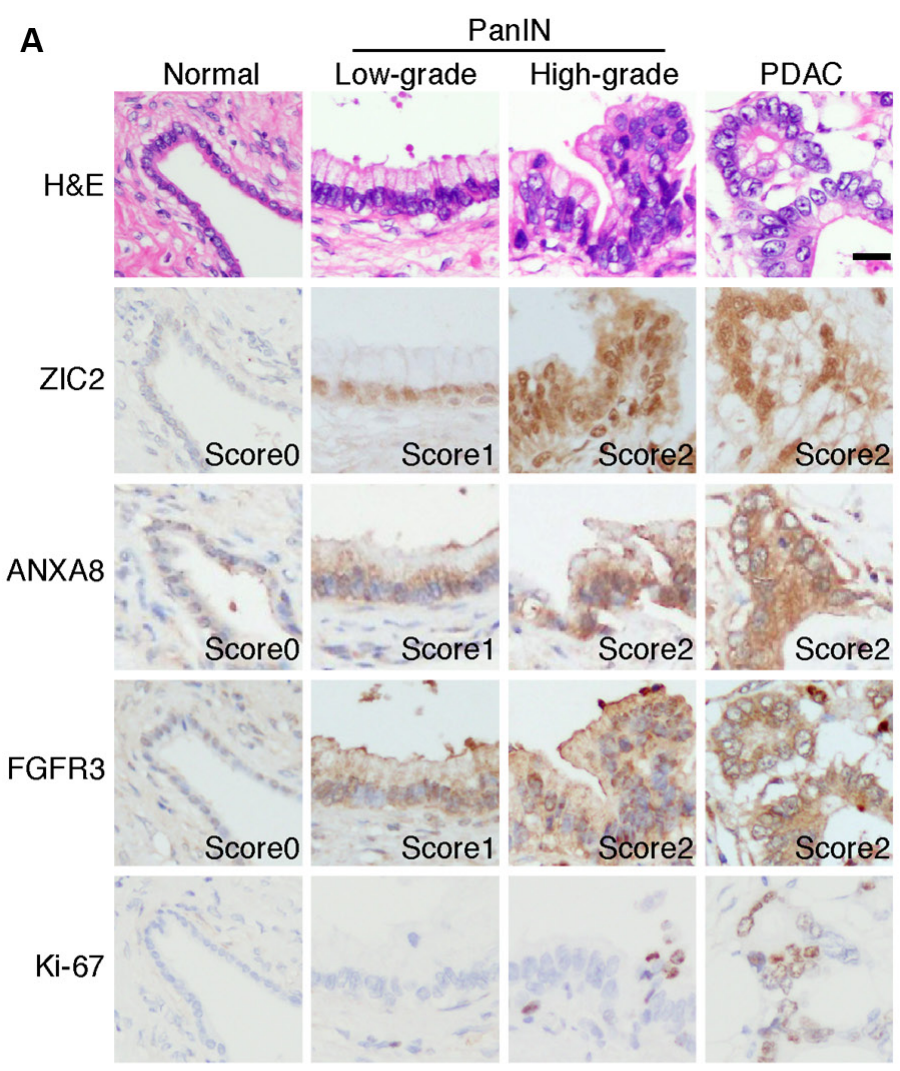

Bar: $20 \mu \mathrm{m}$

B

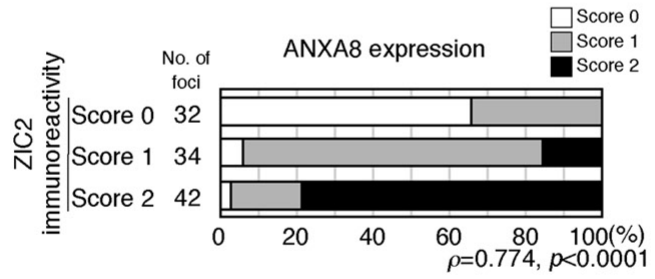

C

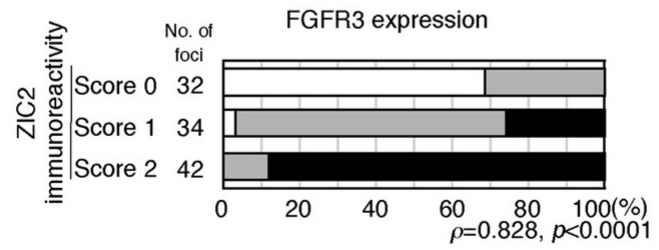

D

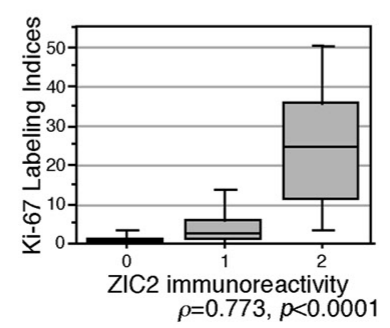

Figure 6: Immunohistochemical analysis of the pancreas harboring PDAC. A, H\&E and immunohistochemical staining of precancerous pancreatic intraepithelial lesion (PanIN) and PDAC. Staining intensities of ZIC2, ANXA8 and FGFR3 were semiquantitatively scored as negative, 0 ; weak, 1 ; strong, 2. Labeling index of Ki-67 staining was calculated by counting over 100 cells per

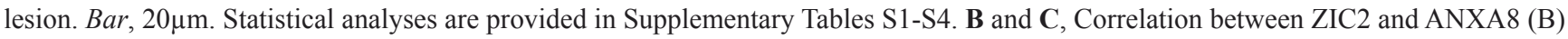
or FGFR3 (C) expression. D, Correlation between ZIC2 expression and the Ki-67 labeling index. 
However, knowledge regarding the role of ZIC in cancer is limited: ZIC1 was reported as a tumor -suppressor in gastric [30] and colorectal cancers [31] because of its down-regulated or silenced expression due to the promoter hypermethylation, while another group showed that $\mathrm{ZIC1}$ is over-expressed and plays an oncogenic role in liposarcoma [32]. ZIC2 expression was revealed to correlate with a worse clinical course of ovarian cancer [18] and oral cancer patients [20], while the molecular mechanism of ZIC2 expression in adult tumor cells and worse prognosis were not fully understood.

In the present study, we revealed that $\mathrm{ZIC} 2$ is uniquely expressed in PDAC cells and regulated the cellular proliferation and apoptotic cell death of PDAC cells through the up-regulation of FGFR3 and $A N X A 8$ expression. Furthermore, using GLI high- and lowexpressing PDAC cell lines, we revealed that the upregulation of those genes occured in a GLI-independent manner. This evidence highlights the unique role of ZIC2 in the regulation of PDAC cell apoptosis.

In a variety of human malignancies, FGFR signaling is activated by several molecular mechanisms [21]. For instance, some cases of bladder cancer harbor the mutated FGFR3 gene, leading to the ligand-independent dimerization or enhanced kinase activity of FGFR3 [28]. In the present study, we found that the forced expression of ZIC2 induced the up-regulation of both splice variants of FGFR3 (FGFR3-IIIb and IIIC) (Figure 3B) and that $F G F R 3$ knockdown induced apoptotic cell death in both the "epithelial" and "mesenchymal" types of PDAC (Figure 5C). Therefore, we currently speculate that a physiological or "ZIC2-inducible" level of FGFR3 might contribute the regulation of apoptotic cell death, even in the "epithelial" type of PDAC, or that the combined upregulation with $A N X A 8$ can prevent the suppressive effect of overexpressed FGFR3 on the cell proliferation of the "epithelial" type of PDAC.

$A N X A 8$ belongs to a vertebrate "A subgroup" of the annexin superfamily coding a calcium- and membranebinding protein. The "A subgroup" consists of at least twelve members (A1-A11and A13), all of which are suspected to be involved in tumor development [22]. ANXA8 has been reported to be up-regulated in BRCA1related breast cancer [33] and PDAC [23]. It was also revealed that that the forced expression of $A N X A 8$ increased the BrdU incorporation of PANC-1 cells and $A N X A 8$ knockdown conversely induced apoptotic cell death [22]; however, the regulatory mechanism of $A N X A 8$ expression is unknown. Here, we showed that $A N X A 8$ as well as FGFR3 were downstream of ZIC2 in PDAC cells. Unfortunately, we failed to identify the ZIC2-binding site(s) within up to $4 \mathrm{~kb}$ DNA fragment of the 5' flanking sequence from transcription start sites of the $A N X A 8$ and FGFR3 genes. Recently, Luo et al. performed ZIC2 chromatin immunoprecipitation and sequence (ChIPseq) analysis in mouse ES cells and revealed that ZIC2 occupied enhancers, the majority of which, however, did not overlap with a transcription start site and instead resided within a gene or upstream / downstream of the gene [25]. This report might suggest the possibility of the presence of the ZIC2-binding site(s) outside of the genomic region that we examined in the present study.

To the best of our knowledge, the mechanism(s) underlying ZIC2-overexpression in cancer cells is currently unknown, which should be investigated in the future.

In conclusion, our evidence uncovered the indispensable and unique role of ZIC2 in PDAC in regulating cell proliferation and apoptotic cell death via GLI-independent downstream genes, underlining ZIC2 as a potential therapeutic target in PDAC patients.

\section{MATERIALS AND METHODS}

\section{Cells, plasmid vectors and siRNAs}

The immortalized normal pancreatic epithelial cells, hTERT-HPNE cells, were purchased from the ATCC. The origins of the other human PDAC cell lines and the methods for their authentication were previously described [10]. PANC-1 ${ }^{\text {Tet/ZIC2 }}$ clone1, clone2 and their control PANC-1 $1^{\text {Tet/empty }}$ were established from human PDAC cell line PANC-1 using a tetracycline-regulated (Tet-off) system according to the manufacturer's instructions (Clontech). Doxycycline (DOX) was used as a regulator of the Tet-off system. The expression vectors for the chimeric gene ZIC2ER and its control ER, ANXA8 and FGFR3 were generated by PCR -amplification and cloning into the pcDNA 3.1 expression vector (Invitrogen). The plasmid vector for the construction of FLGA-ZIC2 and LacZ-expressing lentivirus (CSII-CMVMCS-IRES2-Bsd) was kindly gifted by Dr. Hiroyuki Miyoshi (RIKEN BRC, Japan). To knock -down ZIC2, ANXA8 and FGFR3, 21-nucleotide duplex siRNAs were synthesized as follows (Nippon Gene, Japan): sizIC2-1, 5'-GAAGAGCUGCAACAAAACUTT-3' and 5'-AGUUUUGUUGCAGCUCUUCTT-3'; siZIC2-2, 5'-GUGCGAGUUUGAGGGCUGUTT-3' and 5'-ACAGCCCUCAAACUCGCACTT-3'; siANXA8-1, 5'-CCCAAAACCUCCACAGCUATT-3' and 5'-UAGCUGUGGAGGUUUUGGGTT-3'; siANXA8-2, 5'-AGGAGGGUGUCAUCAUUGATT-3' and 5'-UCAAUGAUGACACCCUCCUTT-3'; siFGFR3-1, 5'-CACCCUACGUUACCGUGCUTT-3' and 5'-AGCACGGUAACGUAGGGUGTT-3'; and siFGFR3-2, 5'-ACUGCACACACGACCUGUATT-3' and 5'-UACAGGUCGUGUGUGCAGUT-3'. The following duplexes were used as a control: siControl 5'-GACAACGACGAAAGAUACUTT-3' and 5'-AGUAUCUUUCGUCGUUGUCTT-3'. The siRNAs 
for GLI1 (siGLI1) and GLI2 (siGLI2) were previously reported [34].

\section{Antibodies and real-time PCR analyses}

The antibodies used were as follows: anti-ZIC2 antibody (ARP35821, Aviva Systems Biology); antiGLI2 antibody (sc-271786; Santa Cruz Biotechnology), anti-p-FGFR-3 (Tyr724) antibody (sc-33041, Santa Cruz Biotechnology); anti-GLI1 L42B10 antibody, anti-FGFR3 C51F2 antibody, anti-p44/42 MAPK 137F5 antibody, antiphospho-p44/42 MAPK 20G11 antibody, anti-cleaved PARP D64E10 antibody (Cell Signaling); anti-FLAG M2 antibody, anti- $\beta$-actin AC-74 antibody (SIGMA); and anti-ANXA8 antibody (GTX103853, GenTex). The methods for the real-time PCR (qRT-PCR) analyses were previously described [10]. All data were analyzed in triplicate.

\section{cDNA microarray analysis}

Total RNA was purified using the RNeasy mini kit (Qiagen) from PANC-1 ${ }^{\text {Tet/ZIC2 }}$ clone1 and PANC-1 $1^{\text {Tet/empty }}$ before and 48 hours after DOX elimination. Total RNA was also extracted from PANC-1 cells transfected with either siZIC2-1 or siControl siRNA for 96 hours. Gene expression was analyzed using Agilent 4x44K cDNA microarray (Agilent Technologies) according to the manufacturer's protocol. Microarray data are available from the NCBI Gene Expression Omnibus (GEO) database (GSE39704).

\section{Cell proliferation and FACS analysis}

To quantify cell numbers, Cell Titer 96(R) Aqueous One Solution (Promega) was used. Cell cycle distributions were analyzed using a Click-iT ${ }^{\circledR}$ EdU Alexa Fluor ${ }^{\circledR}$ 488 Flow Cytometry Assay Kit (Life Technologies) or propidium iodide (PI) staining. The stained cells were counted using FACSCanto II flow cytometer (BectonDickinson). All data were analyzed in triplicate.

\section{Anchorage-independent growth analysis}

Lentiviral-transduced HPNE cells were cultured in a soft -agar. The number of cells in the soft -agar was measured using a Cytoselect 96-well cell transformation assay (Cell Biolabs Inc., USA) according to the manufacturer's protocol.

\section{Immunohistochemical analysis}

Twenty PDAC cases were selected for the study based on the availability of tissue samples from the archives of the Department of Pathology at the Aichi Medical University Hospital. Sample use was approved by the Institutional Ethical Review Board. Serial sections from formalin-fixed, paraffin-embedded tissue samples were subjected to the hematoxylin and eosin (H\&E) staining and immunohistochemical staining. The staining intensities of ZIC2, ANXA8 and FGFR3 were semi-quantitatively scored using a three-tiered scale and statistically analyzed using a Mann-Whitney $U$ test. For the analysis of Ki-67 staining, a labeling index was calculated by counting at least one hundred of cells in the lesion. The correlations between ZIC2, ANXA8, FGFR3 expressions and Ki-67 labeling index were estimated using Spearman's correlation coefficient. P-values of $<0.05$ were considered statistically significant. StatView 5.0 (SAS Institute Inc.) was used for statistical analyses.

\section{ACKNOWLEDGMENTS}

We thank Michiko Ishikawa, Motoyasu Takeuchi, Naoki Igari and Yukiko Matsubara (Aichi Medical University) for assistance with immunohistochemical staining and Dr. Hiroyuki Miyoshi (RIKEN BRC) for the lentiviral vector.

\section{GRANT SUPPORT}

This study was supported in part by JSPS KAKENHI Grant Numbers 21790370, 23790424 (to SI), 24590456 (to KK) from the Japan Society for the Promotion of Science, and Grants-in-Aid from the Pancreas Research Foundation of Japan (to SI and HI), Aikeikai Foundation (to SI and HI) and Aichi Cancer Research Foundation (to KK).

\section{CONFLICTS OF INTEREST}

No potential conflict of interest are disclosed.

\section{REFERENCES}

1. Grinberg I and Millen KJ. The ZIC gene family in development and disease. Clin Genet. 2005; 67:290-296.

2. Mizugishi K, Aruga J, Nakata K and Mikoshiba K. Molecular properties of Zic proteins as transcriptional regulators and their relationship to GLI proteins. J Biol Chem. 2001; 276:2180-2188.

3. Cohen MM, Jr. Hedgehog signaling update. Am J Med Genet A. 2010; 152A:1875-1914.

4. Barakat MT, Humke EW and Scott MP. Learning from 
Jekyll to control Hyde: Hedgehog signaling in development and cancer. Trends Mol Med. 2010; 16:337-348.

5. Nolan-Stevaux O, Lau J, Truitt ML, Chu GC, Hebrok M, Fernandez-Zapico ME and Hanahan D. GLI1 is regulated through Smoothened-independent mechanisms in neoplastic pancreatic ducts and mediates PDAC cell survival and transformation. Genes Dev. 2009; 23:24-36.

6. Pasca di Magliano M, Sekine S, Ermilov A, Ferris J, Dlugosz AA and Hebrok M. Hedgehog/Ras interactions regulate early stages of pancreatic cancer. Genes Dev. 2006; 20:3161-3173.

7. Rajurkar M, De Jesus-Monge WE, Driscoll DR, Appleman VA, Huang H, Cotton JL, Klimstra DS, Zhu LJ, Simin K, $\mathrm{Xu}$ L, McMahon AP, Lewis BC and Mao J. The activity of Gli transcription factors is essential for Kras-induced pancreatic tumorigenesis. Proc Natl Acad Sci U S A. 2012; 109:E1038-1047.

8. Eichberger T, Sander V, Schnidar H, Regl G, Kasper M, Schmid C, Plamberger S, Kaser A, Aberger F and Frischauf AM. Overlapping and distinct transcriptional regulator properties of the GLI1 and GLI2 oncogenes. Genomics. 2006; 87:616-632.

9. Inaguma $\mathrm{S}$, Kasai $\mathrm{K}$ and Ikeda H. GLI1 facilitates the migration and invasion of pancreatic cancer cells through MUC5AC-mediated attenuation of E-cadherin. Oncogene. 2011; 30:714-723.

10. Inaguma $\mathrm{S}$, Riku $\mathrm{M}$, Hashimoto $\mathrm{M}$, Murakami H, Saga $\mathrm{S}$, Ikeda $\mathrm{H}$ and Kasai K. GLI1 interferes with the DNA mismatch repair system in pancreatic cancer through BHLHE41-mediated suppression of MLH1. Cancer Res. 2013; 73:7313-7323.

11. Kasper M, Schnidar H, Neill GW, Hanneder M, Klingler S, Blaas L, Schmid C, Hauser-Kronberger C, Regl G, Philpott MP and Aberger F. Selective modulation of Hedgehog/GLI target gene expression by epidermal growth factor signaling in human keratinocytes. Mol Cell Biol. 2006; 26:62836298.

12. Yoon JW, Kita Y, Frank DJ, Majewski RR, Konicek BA, Nobrega MA, Jacob H, Walterhouse D and Iannaccone P. Gene expression profiling leads to identification of GLI1binding elements in target genes and a role for multiple downstream pathways in GLI1-induced cell transformation. J Biol Chem. 2002; 277:5548-5555.

13. Chan DW, Liu VW, Leung LY, Yao KM, Chan KK, Cheung AN and Ngan HY. Zic2 synergistically enhances Hedgehog signalling through nuclear retention of Gli1 in cervical cancer cells. J Pathol. 2011; 225:525-534.

14. Koyabu Y, Nakata K, Mizugishi K, Aruga J and Mikoshiba K. Physical and functional interactions between Zic and Gli proteins. J Biol Chem. 2001; 276:6889-6892.

15. Badea L, Herlea V, Dima SO, Dumitrascu T and Popescu I. Combined gene expression analysis of whole-tissue and microdissected pancreatic ductal adenocarcinoma identifies genes specifically overexpressed in tumor epithelia.
Hepatogastroenterology. 2008; 55:2016-2027.

16. Grutzmann R, Pilarsky C, Ammerpohl O, Luttges J, Bohme A, Sipos B, Foerder M, Alldinger I, Jahnke B, Schackert HK, Kalthoff H, Kremer B, Kloppel G and Saeger HD. Gene expression profiling of microdissected pancreatic ductal carcinomas using high-density DNA microarrays. Neoplasia. 2004; 6:611-622.

17. Pei H, Li L, Fridley BL, Jenkins GD, Kalari KR, Lingle W, Petersen G, Lou Z and Wang L. FKBP51 affects cancer cell response to chemotherapy by negatively regulating Akt. Cancer Cell. 2009; 16:259-266.

18. Marchini S, Poynor E, Barakat RR, Clivio L, Cinquini M, Fruscio R, Porcu L, Bussani C, D’Incalci M, Erba E, Romano M, Cattoretti G, Katsaros D, Koff A and Luzzatto L. The zinc finger gene ZIC2 has features of an oncogene and its overexpression correlates strongly with the clinical course of epithelial ovarian cancer. Clin Cancer Res. 2012; 18:4313-4324.

19. Bidus MA, Risinger JI, Chandramouli GV, Dainty LA, Litzi TJ, Berchuck A, Barrett JC and Maxwell GL. Prediction of lymph node metastasis in patients with endometrioid endometrial cancer using expression microarray. Clin Cancer Res. 2006; 12:83-88.

20. Sakuma K, Kasamatsu A, Yamatoji M, Yamano Y, Fushimi K, Iyoda M, Ogoshi K, Shinozuka K, Ogawara K, Shiiba M, Tanzawa $\mathrm{H}$ and Uzawa K. Expression status of Zic family member 2 as a prognostic marker for oral squamous cell carcinoma. J Cancer Res Clin Oncol. 2010; 136:553-559.

21. Wesche J, Haglund K and Haugsten EM. Fibroblast growth factors and their receptors in cancer. Biochem J. 2011; 437:199-213.

22. Mussunoor S and Murray GI. The role of annexins in tumour development and progression. J Pathol. 2008; 216:131-140.

23. Karanjawala ZE, Illei PB, Ashfaq R, Infante JR, Murphy K, Pandey A, Schulick R, Winter J, Sharma R, Maitra A, Goggins $M$ and Hruban RH. New markers of pancreatic cancer identified through differential gene expression analyses: claudin 18 and annexin A8. Am J Surg Pathol. 2008; 32:188-196.

24. Hata $H$, Tatemichi $M$ and Nakadate T. Involvement of Annexin A8 in the properties of pancreatic cancer. Mol Carcinog. 2014;53:181-191.

25. Luo Z, Gao X, Lin C, Smith ER, Marshall SA, Swanson SK, Florens L, Washburn MP and Shilatifard A. Zic2 is an enhancer-binding factor required for embryonic stem cell specification. Mol Cell. 2015; 57:685-694.

26. Lafitte M, Moranvillier I, Garcia S, Peuchant E, Iovanna J, Rousseau B, Dubus P, Guyonnet-Duperat V, Belleannee G, Ramos J, Bedel A, de Verneuil H, Moreau-Gaudry F and Dabernat S. FGFR3 has tumor suppressor properties in cells with epithelial phenotype. Mol Cancer. 2013; 12:83. doi: 10.1186/1476-4598-12-83.

27. Furukawa T. Impacts of activation of the mitogen-activated 
protein kinase pathway in pancreatic cancer. Front Oncol. 2015; 5:23. doi: 10.3389/fonc.2015.00023

28. Iyer $G$ and Milowsky MI. Fibroblast growth factor receptor-3 in urothelial tumorigenesis. Urol Oncol. 2013; 31:303-311.

29. Kasai K, Inaguma S, Yoneyama A, Yoshikawa K and Ikeda H. SCL/TAL1 interrupting locus derepresses GLI1 from the negative control of suppressor-of-fused in pancreatic cancer cell. Cancer Res. 2008; 68:7723-7729.

30. Wang LJ, Jin HC, Wang X, Lam EK, Zhang JB, Liu X, Chan FK, Si JM and Sung JJ. ZIC1 is downregulated through promoter hypermethylation in gastric cancer. Biochem Biophys Res Commun. 2009; 379:959-963.

31. Gan L, Chen S, Zhong J, Wang X, Lam EK, Liu X, Zhang J, Zhou T, Yu J, Si J, Wang L and Jin H. ZIC1 is downregulated through promoter hypermethylation, and functions as a tumor suppressor gene in colorectal cancer. PLoS One. 2011; 6:e16916. doi: 10.1371/journal. pone. 0016916 .

32. Brill E, Gobble R, Angeles C, Lagos-Quintana M, Crago A, Laxa B, Decarolis P, Zhang L, Antonescu C, Socci ND, Taylor BS, Sander C, Koff A and Singer S. ZIC1 overexpression is oncogenic in liposarcoma. Cancer Res. 2010; 70:6891-6901.

33. Stein T, Price KN, Morris JS, Heath VJ, Ferrier RK, Bell AK, Pringle MA, Villadsen R, Petersen OW, Sauter G, Bryson G, Mallon EA and Gusterson BA. Annexin A8 is up-regulated during mouse mammary gland involution and predicts poor survival in breast cancer. Clin Cancer Res. 2005; 11:6872-6879.

34. Inaguma $\mathrm{S}$, Kasai $\mathrm{K}$, Hashimoto $\mathrm{M}$ and Ikeda H. GLI1 modulates EMT in pancreatic cancer--letter. Cancer Res. 2012; 72:3702-3703; author reply 3704-3705. 